\title{
Assessment of awareness about teledentistry among dentists in a private dental college of Muradnagar, Ghaziabad
}

\author{
Deepa Tomar ${ }^{1, *}$, Ipseeta Menon ${ }^{2}$, Upasana Tyagi ${ }^{3}$, Avnish Singh $^{4}$, Jyoti Goyal ${ }^{5}$, Ricky Pal Singh $^{6}$, Anubhav \\ Sharma ${ }^{7}$ \\ ${ }^{1,3,5}$ PG Student, ${ }^{2,4}$ Reader, ${ }^{6,7}$ Senior Lecturer, Dept. of Public Health Dentistry, I.T.S Centre for Dental Studies \& Research, \\ Muradnagar, Ghaziabad, Uttar Pradesh, India
}

*Corresponding Author:

Email: drtomar99999@gmail.com

\begin{abstract}
Aim: Assessment of awareness about teledentistry among dentists in a private dental college of Muradnagar, Ghaziabad.

Materials and Method: A total of 350 dental professionals of a private dental college were selected for the study. A selfadministered validated questionnaire was prepared or the assessment of awareness regarding teledentistry among dental professionals of different academic qualification. SPSS software version 18 was used for statistical analysis.

Results: $41 \%$ of the students were males. $48 \%$ of study participants belong to $<25$ years of age group.

Conclusion Awareness must be spread among the dental professionals regarding the proper use of teledentistry in future practice.
\end{abstract}

Keywords: Teledentistry, Knowledge, Private dental college, Dentists.

\section{Introduction}

Data and innovation are assuming a crucial part in changing dentistry in this cutting- edge period. Teledentistry can be understood as the act of utilizing video-conferencing advancements to analyze and give counsel about treatment over a distance.

Teledentistry is an extension of Telemedicine. Tele in Greek means distance and mederi in Latin means to heal. ${ }^{1}$ Teledentistry has numerous branches like Telestomatology, Teleradiology, Telepathology, Tele oral medical procedure and Teleorthodontics. Teledentistry, a sprouting field of dentistry, utilizes data based advancements and correspondence frameworks to convey health to the general population. The essential arrangement of dental care data is brought out through the best possible channels of advanced correspondence. It can be a conspicuous hotspot for entombing proficient correspondences and in the long run can be utilized for better instructive source among dental graduates. It additionally helps in granting essential information to patients about dental and medicinal services.

Oral wellbeing is an unavoidable part of general wellbeing. Teledentistry can be utilized as a part of each branch of dentistry. The mix of innovation with dentistry frames teledentistry. The data was traded amongst patient and specialist and in addition to different authorities through electronic devices over remote separations for a superior treatment need and result. Most teledentistry projects to date have centered upon separate administration and organization of remote offices, learning and proceeding with instruction, and interview and referral benefits as opposed to the supervision of helpers or direct patient care. Teledentistry can be utilized for instructing postgraduate students and refreshing the dental practitioners.

Teledentistry plays a vital role in various fields of dentistry. In Oral Medicine and Radiology, it helps in diagnosing and framing a treatment plan for difficult cases by easy access to various specialists through the transfer of radiologic images of lesions. In maxillofacial surgery, teledentistry may be helpful for appropriate treatment of complicated cases by analysis of advanced dental imaging techniques (like CBCT) which are often not available in one centre. ${ }^{2}$ While in Orthodontics it causes an extraordinary arrangement to take various sentiments on a routine cephalometric investigation for a superior treatment result. Additionally, teledentistry can be utilized for better patient consistency and fulfillment by sharing pre and post treatment pictures. In Endodontics, it can be appropriate for an exact analysis of periapical sore and nearness of any supplementary root trench. In Prosthodontics, teledentistry alongside different computerized analytic devices (like CAD, CAM) can be a valuable instrument in outlining on lays and crown planning also.

In interactive video-conferencing, the patient data is assessed first (with or without the patient's presence), which takes into consideration the communication and input between the instructor and the understudies. The patient cases assessed altogether and at the understudies' pace. The cases can be talked about finally after all the clinical information have been gathered and transmitted, without the patient is available at the booked gathering. This upgrades the understudies' energy and gives new learning chances to the dental graduates.

To incorporate the knowledge and practice of teledentistry in the future generation dentists it is important to assess the awareness of the present dental 
students. Hence, this study was done to assess the awareness about teledentistry among dentists in a private dental college of Muradnagar, Ghaziabad.

\section{Materials and Method}

A cross-sectional survey was conducted among dental professionals in a private dental college, to know the awareness of teledentistry among dental professionals. Before starting the study ethical clearance was obtained from the ethical committee of ITS-CDSR Muradnagar. Written consent from dental professionals was taken before their participation in the study.

50 dental professionals from $1^{\text {st }}$ year BDS, 50 from $2^{\text {nd }}$ year, 50 from $3^{\text {rd }}$ year, 50 from 4 th year BDS, 50 from interns, 50 from postgraduate students and 50 from staff members were randomly selected from the dental college on the day of data collection which ultimately gives us a total of 350 randomly selected dental professionals on the day of data collection from the dental college. A questionnaire was prepared to assess the knowledge of teledentistry among dental professionals and was circulated among dental professionals in the dental college. The questionnaire consisted of 12close-ended, validated, structured, selfadministered questions on awareness regarding teledentistry. The mean Kappa coefficient values for intra-examiner reliability was 0.88 .

On the pre-decided days, investigator circulated the questionnaire. The completed questionnaire was collected and subjected to statistical analysis with SPSS software 18.0. The forms were arranged in serial number and stacked together. The bundles were labeled with ID numbers and date of recording so as to make them ready for data entry. The data entry was made on the same day so that if any discrepancy was seen it could be rectified easily. The survey forms were rechecked to assess any missing information. Statistical analysis was done by SPSS 18.0. Data was compiled and the master table was prepared. Meaningful distribution and presentation of data were done. Descriptive statistical analysis of data had been carried out in the present study.

\section{Results}

A total of 350 persons responded to the questionnaire. $59 \%$ were females and $41 \%$ were males among the 350 participated subjects in the study (Fig 1). $10 \%$ of study subjects were of $>35$ years, $42 \%$ were of 25 to 35 years, $46 \%$ were of $<25$ years (Fig. 2). Maximum number of study population uses internet for 0-2 hours i.e. $52 \%$ followed by $2-4$ hours by $40 \%$ and $8 \%$ study population uses internet for 4-6 hours in a day on daily basis (Fig. 3).

Table 1 shows the knowledge and awareness of the study subjects of various qualifications about teledentistry. In this study staff members were having maximum awareness about teledentistry (84.18\%). Most of the study population didn't know how to use teledentistry in future but most of them want to use teledentistry in day to day practice.

Most of the investigation populace concurred with that the teledentistry is constrained to dentistry however few of the examination populace won't be used in future as a result of its excessively numerous legitimate issues as indicated by them.

The greater part of members concurred that teledentistry can truly help in wellbeing instruction of masses and most extreme were from staff $(86.32 \%)$. Less number of study populace felt that teledentistry isn't valuable in wellbeing instruction of group. The greater part of members imagined that teledentistry can be a decent instrument for oral cleanliness preparing. Over half respondents have concurred that teledentistry isn't a financially savvy alternative. The dominant part of the members were supportive of the way that teledentistry is efficient if fused in every day clinical practice.

$94.00 \%$ of staff members and $88.10 \%$ postgraduate students agreed that teledentistry can be applied in every branch of dentistry. Most of the dentists believed that teledentistry can be standard way of oral health delivery and the maximum of 3rd year BDS students and only $40.50 \%$ staff members thought that teledentistry can be used as a standard oral health care delivery system.

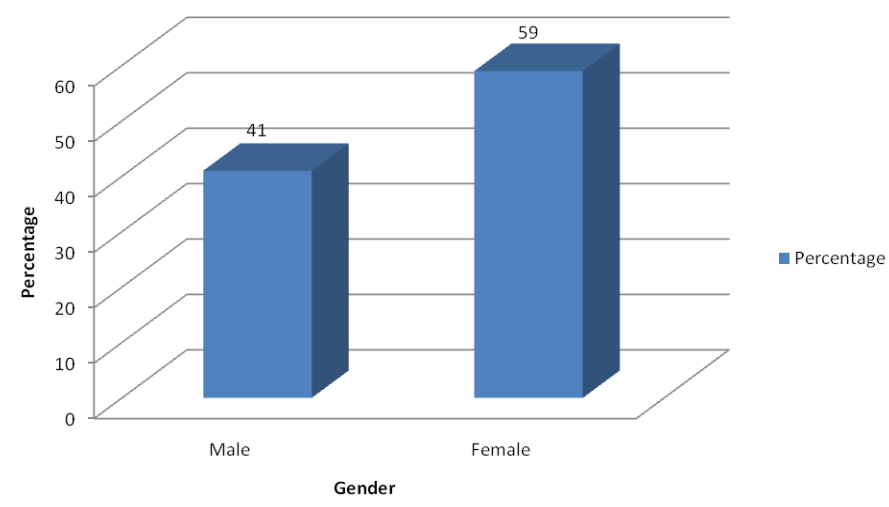


Fig. 1: Distribution of study population according to gender

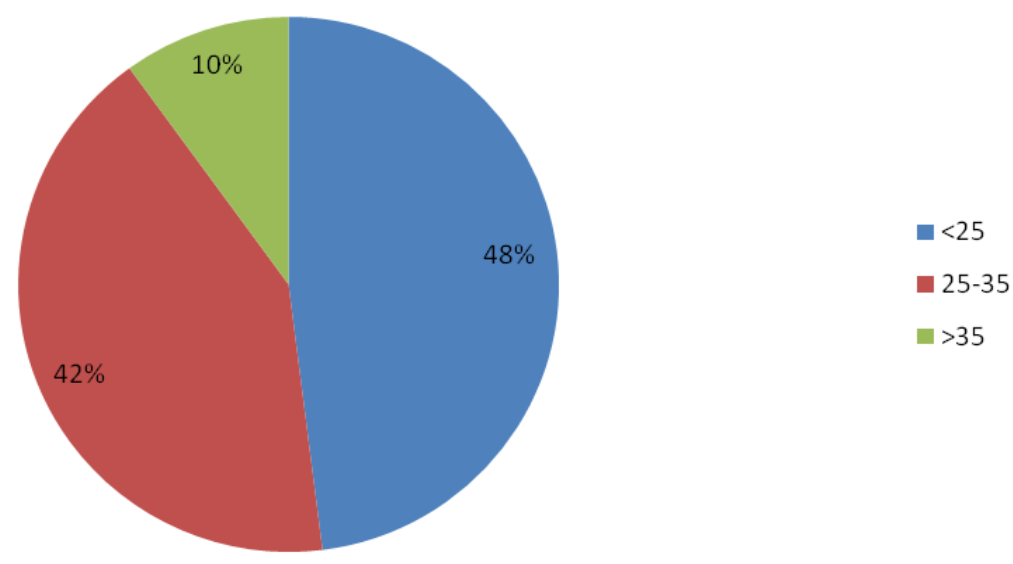

Fig. 2: Distribution of study population according to age

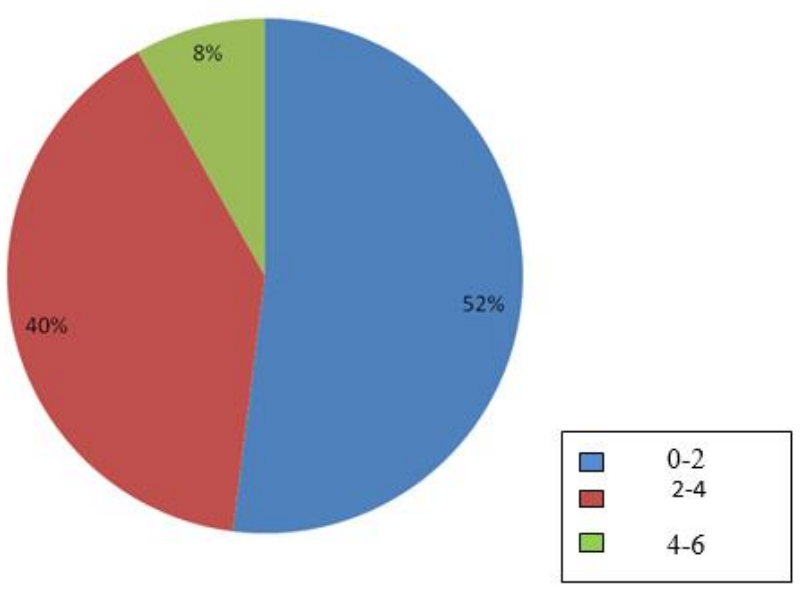

Fig. 3: Distribution of study subject according to internet access (in hours)

Table 1: Showing responses of awareness of teledentistry among the study population of various academic qualification

\begin{tabular}{|c|c|c|c|c|c|c|c|}
\hline Questions & $\begin{array}{c}1^{\text {st }} \text { year } \\
\text { BDS }\end{array}$ & $\begin{array}{c}2^{\text {nd }} \text { year } \\
\text { BDS }\end{array}$ & $\begin{array}{c}3^{\text {rd }} \text { year } \\
\text { BDS }\end{array}$ & $\begin{array}{l}4^{\text {th }} \text { year } \\
\text { BDS }\end{array}$ & Interns & PG & Staff \\
\hline \multicolumn{8}{|c|}{ 1. Have you heard about teledentistry? } \\
\hline Yes & 36.65 & 32.33 & 39.53 & 35.20 & 62.98 & 77.21 & 84.18 \\
\hline \multicolumn{8}{|c|}{ 2. What do you mean by teledentistry? } \\
\hline \multicolumn{8}{|l|}{ Through telephone } \\
\hline Media & 33.54 & 32.02 & 35.63 & 34.30 & 59.48 & 65.22 & 83.43 \\
\hline \multicolumn{8}{|c|}{ 3. In future will you practice teledentistry? } \\
\hline Yes & 33.23 & 46.37 & 43.35 & 53.34 & 67.62 & 63.86 & 83.51 \\
\hline \multicolumn{8}{|c|}{ 4. What is your opinion about teledentistry? } \\
\hline Very useful & 25.49 & 25.12 & 39.00 & 15.29 & 22.80 & 04.00 & 20.41 \\
\hline $\begin{array}{l}\text { Limited in general } \\
\text { medicine }\end{array}$ & 19.44 & 28.88 & 15.09 & 21.59 & 32.14 & 6.84 & 15.20 \\
\hline Limited in dentistry & 46.94 & 40.20 & 43.42 & 50.62 & 35.06 & 85.14 & 57.24 \\
\hline Too many legal issues & 8.13 & 5.80 & 2.49 & 12.50 & 10.00 & 04.02 & 7.15 \\
\hline \multicolumn{8}{|c|}{ 5. Do you agree that teledentistry can be a good tool for oral hygiene training? } \\
\hline Yes & 54.35 & 44.33 & 43.11 & 46.23 & 52.25 & 60.00 & 75.00 \\
\hline \multicolumn{8}{|c|}{ 6. Do you agree that teledentistry is not financially feasible? } \\
\hline Yes & 49.99 & 49.22 & 50.93 & 57.53 & 66.46 & 70.01 & 78.91 \\
\hline
\end{tabular}




\begin{tabular}{|c|c|c|c|c|c|c|c|}
\hline \multicolumn{8}{|c|}{ 7. Do you think teledentistry is time saving? } \\
\hline Yes & 48.26 & 42.78 & 56.74 & 56.09 & 69.78 & 70.40 & 62.84 \\
\hline \multicolumn{8}{|c|}{ 8. Teledentistry can be applied in every branch of dentistry? } \\
\hline Yes & 66.63 & 56.76 & 70.84 & 58.26 & 68.55 & 88.10 & 94.00 \\
\hline \multicolumn{8}{|c|}{ 9. Teledentistry will help to consult with an expert about specific patient`s problem? } \\
\hline Yes & 44.31 & 53.35 & 59.44 & 76.29 & 77.85 & 81.60 & 87.62 \\
\hline \multicolumn{8}{|c|}{$\begin{array}{l}\text { 10. Teledentistry can provide me a good understanding of the patient's oral health problem over the } \\
\text { internet? }\end{array}$} \\
\hline Yes & 48.0 & 60.11 & 30.04 & 40.90 & 51.67 & 70.30 & 56.21 \\
\hline \multicolumn{8}{|c|}{ 11. Teledentistry will be a standard way of oral health care delivery? } \\
\hline Yes & 62.75 & 55.65 & 73.11 & 48.52 & 52.50 & 35.12 & 40.50 \\
\hline \multicolumn{8}{|c|}{ teledentistry can really help in health education of masses? } \\
\hline Yes & 42.25 & 44.15 & 53.45 & 56.59 & 62.31 & 77.14 & 86.32 \\
\hline
\end{tabular}

\section{Discussion}

The present study demonstrates the awareness regarding a new and interesting topic i.e. Teledentistry. It provides an endless potential to the dental professionals by using the internet in dentistry. It also enables the cross-communication between other specialists which helps in better treatment planning. Teledentistry saves time as direct meeting with the patients is not required in it.

In the present study, the knowledge and awareness about teledentistry in staff $(84.18 \%)$ was more as compared to BDS students especially $1^{\text {st }}$ Years BDS (36.65\%).These results were not similar to a study done by Nagarajappa $\mathrm{R}$ et $\mathrm{al}^{3}$ at Udaipur and Boringi ${\mathrm{M} \mathrm{et} \mathrm{al}^{2}}^{2}$ in which the knowledge of $1^{\text {st }}$ year BDS students was more as compared to staff members. The examination likewise plainly demonstrated that dental practitioners with postgraduation speciality were having high mindfulness about tele-dentistry when contrasted with BDS graduates of different scholastic years. The reason might be dental specialists with postgraduate capabilities are more aware of the utilization of computer and web. Utilization of computer and web are basic in human health services segment these days. In an investigation done by Daniel $\mathrm{SJ}^{4}$ clinical practice can be improved by the medium of tele-dentistry.

Over $60 \%$ of the examination members trust that teledentistry is a routine with regards to dentistry in the method of media which is comparative with the investigation done by Chhabra $\mathrm{N}$ et al. ${ }^{5} \mathrm{In}$ the present investigation the vast majority of the staff individuals and not as much as half of the first year BDS students concurred that teledentistry can help in the training of masses. It isn't comparable with an examination done Boringi $\mathrm{M}^{2}$ and by Ata $\mathrm{SO}^{6}$ were $>90 \%$ concurred about tele-eduction. $54.35 \%$ of the first year BDS students concurred that teledentistry can help in better oral hygiene practice drills. This is not exactly as found in an examination done by Nagarajappa $\mathrm{R}$ et $\mathrm{al}^{3}$ where almost $70 \%$ and Boringi $\mathrm{M}$ et al ${ }^{2}$ where about $79.59 \%$ have concurred.

Khan SA, ${ }^{7}$ Ireland $\mathrm{N},{ }^{8}$ and Brullmann $\mathrm{D},{ }^{9}$ looked into that the key techniques utilized as a part of teledentistry are electronic wellbeing records, electronic referral frameworks, digitizing pictures, teleconsultations, and telediagnosis. All these bring proficiency, give access to the underserved population, enhance nature of care, and decrease oral ailment load. Staff individuals trusted that teledentistry is time and cost devouring practice. These outcomes are not comparable with an investigation done by Boringi $\mathrm{M}^{2}$. In another examination done by Ata $\mathrm{SO}^{7}$ in Turkey teledentistry is efficient and an additionally practical approach. As the examination set up was a dental school and healing center henceforth the outcomes can't be summed up in general dental experts.

Most of the dental professionals want to incorporate teledentistry in practice but don't know about the way of implementation so training must be given to them. Various CDE projects and workshops must be composed for dental experts on new advancements in innovation. The overnment must take care of the issue of repayment for dental treatment as installment is the principal impediment in India on the way of teledentistry. The dental universities with a foreordained catchment zone could be perfect spots to fill in as center point locals for teledentistry interview as they incorporate every one of the authorities serving under a typical rooftop. A group of authorities could impart for a couple of hours consistently with the dental specialists/hygienists/patients at the remote clinics ${ }^{4,10}$.

\section{References}

1. Jain A, Bhaskar DJ, Gupta D, Agali C, Gupta V, Karim B. Teledentistry: UpcomingTrend in Dentistry. J Adv Med Dent Scie 2013;1(2):112-5.

2. Boringi M, Waghray S, Lavanya R, Babu DBG, Badam RK, Harsha $\mathrm{N}$ et al, Knowledge and Awareness of Teledentistry among Dental Professionals - A Cross Sectional Study. J Clin Diag Res 2015;9(8):41-4.

3. Nagarajappa R, Aapaliya P, Sharda AJ, Asawa K, Tak M, Pujara P et al. Teledentistry: Knowledge and Attitudes among Dentists in Udaipur, India. Oral Health Dent Manag 2013;12(3):138-44.

4. Daniel SJ, Wu L, Kumar S. Teledentistry: a systematic review of clinical outcomes, utilization and costs. J Dent Hyg 2013;87(6):345-52. 
5. Chhabra N, Chhabra A, Jain RL, Kaur H, Bansal S. Role of Teledentistry in Dental Education: Need of the Era. J Clin Diag Res 2011;5(7):1486-88.

6. Ata SO, Ozkan S. Information technology in oral health care: attitudes of dental professionals on the use of teledentistry in turkey. European and Mediterranean Conference on Information Systems 2009;13-14:1-8.

7. Khan SA, Omar H. Telemed: Teledentistry in practice: literature review. Telecare 2013;19(4):179-83.

8. Ireland N, Bradley M, Black P, Noble S, Thompson R, Lamey R. Application of teledentistry in oral medicine in a community dental service. Br Dent J 2010;209(8):399404.

9. Brullmann D, Schmidtmann I, Warzecha K, Dhoet BJ. Telemed: Recognition of root canal orifices at a distance a preliminary study of teledentistry. Telecare 2011;17(3):154-7.

10. Reddy KV. Using teledentistry for providing the specialist access to rural Indians. Ind J Dent Res 2011;22:189. 